\title{
REACTIVATION OF TUBERCULOSIS AFTER TOTAL HIP REPLACEMENT
}

\author{
ROBERT JOHNSON, K. L. BARNES, R. OWEN
}

\author{
From the Liverpool Royal Infirmary and Wrightington Hospital, Wigan
}

\begin{abstract}
Two cases of reactivation of tuberculous after total hip replacement are reported. Both patients had had tuberculous hips treated without chemotherapy in childhood, and for forty-two and thirty-seven years respectively had had no signs of active infection. Reactivation occurred twelve months after hip replacement. The implications are discussed and preventative measures suggested.
\end{abstract}

Total replacement is now well established as a successful treatment for many abnormalities of the hip. Included among the more difficult cases now being accepted for replacement are those with a history of septic and tuberculous arthritis. The two cases reported here sound a note of caution and are a reminder that tuberculosis can reactivate after prolonged periods (Girdlestone and Somerville 1952).

\section{CASE HISTORIES}

Case 1. This patient had first been seen at the age of thirteen years after a fall and a diagnosis of tuberculosis of the left hip had been made. She had been treated on a frame intermittently for a total of eight years.
She had then been free of symptoms until the age of forty-eight when pain had started in the left hip and had become increasingly severe. She had remained apparently free of infection and at no time had there been any sinuses.

Examination in August 1973 showed that flexion of the hip ranged from zero to 30 degrees, there was fixed adduction of 30 degrees and fixed lateral rotation of 30 degrees with a further jog of lateral rotation. Routine investigations recorded a haemoglobin level of 13.8 grams per 100 millilitres, a white cell count of 5400 per microlitre and an erythrocyte sedimentation rate of 35 millimetres in the first hour. Radiographs suggested a fibrous ankylosis of the left hip (Fig. 1). Operation was deferred for one week because of a coliform infection of the urinary tract, which was treated with Negram. Noother antibiotics were given. A Charnley arthroplasty was then performed on the left hip. She made a good recovery. A culture grown from the

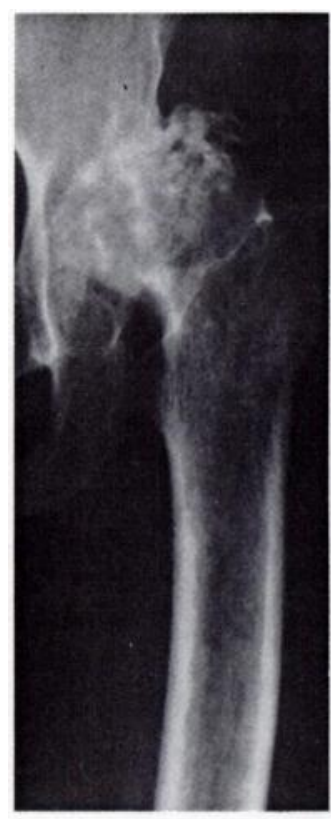

Fig. 1

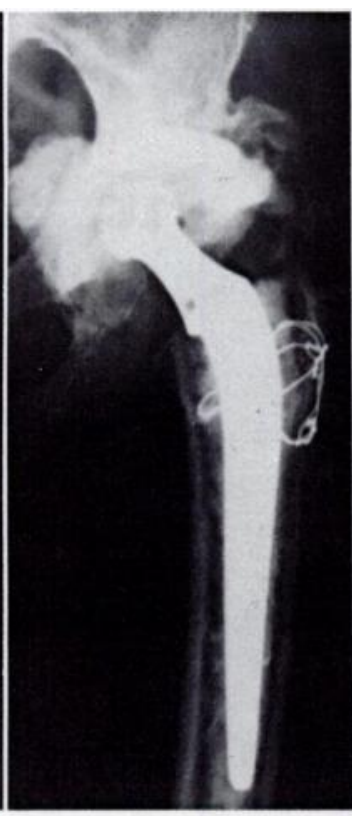

Fig. 2

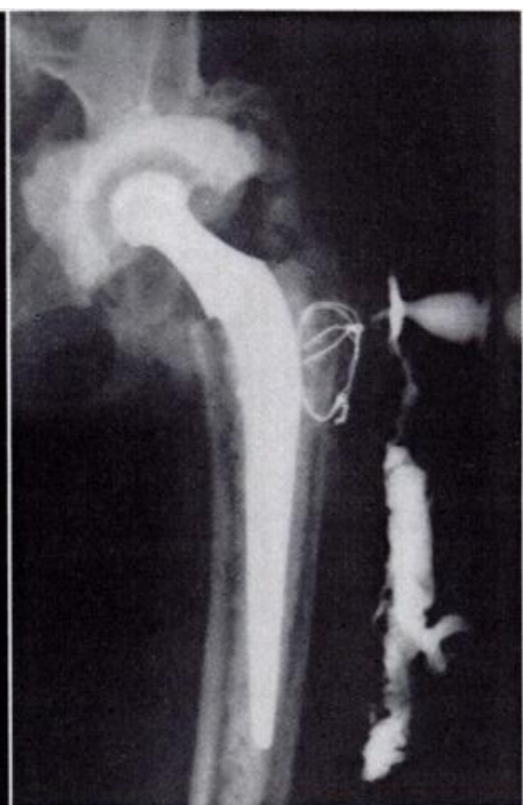

Fig. 3

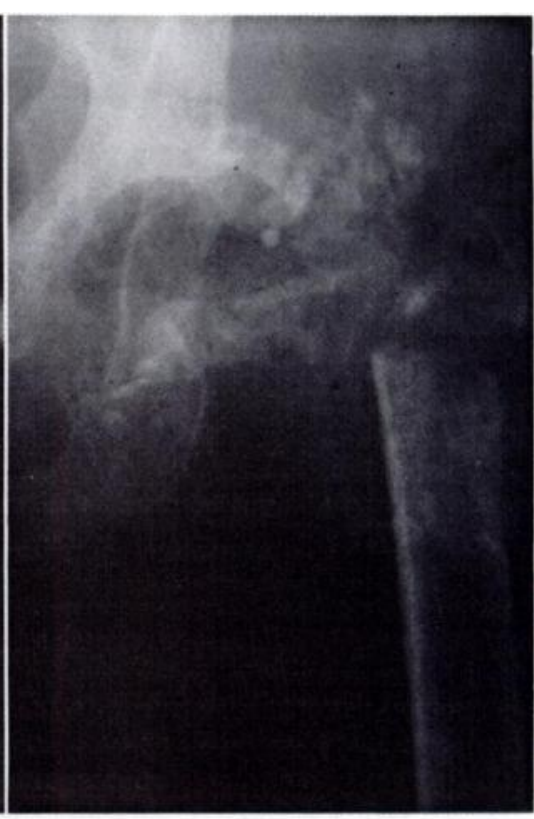

Fig. 4

Case 1. Figure 1-Radiograph before operation. Figure 2-Twelve months after operation. Figure 3-Sinogram two years after operation. Figure 4-After a Girdlestone operation.

R. Johnson, M.Ch.Orth., F.R.C.S., F.R.C.S.Ed.,

Senior Orthopaedic Registrar

R. Owen, M.Ch.Orth., F.R.C.S.

Consultant Orthopaedic Surgeon

Royal Liverpool Hospital, Prescot Street, Liverpool L7 8XP. England.

K. L. Barnes, F.R.C.S., F.R.C.S.Ed., Consultant Orthopaedic Surgeon, Wrightington Hospital, Appley Bridge, near Wigan. England.

Requests for reprints should be sent to Mr R. Johnson. 


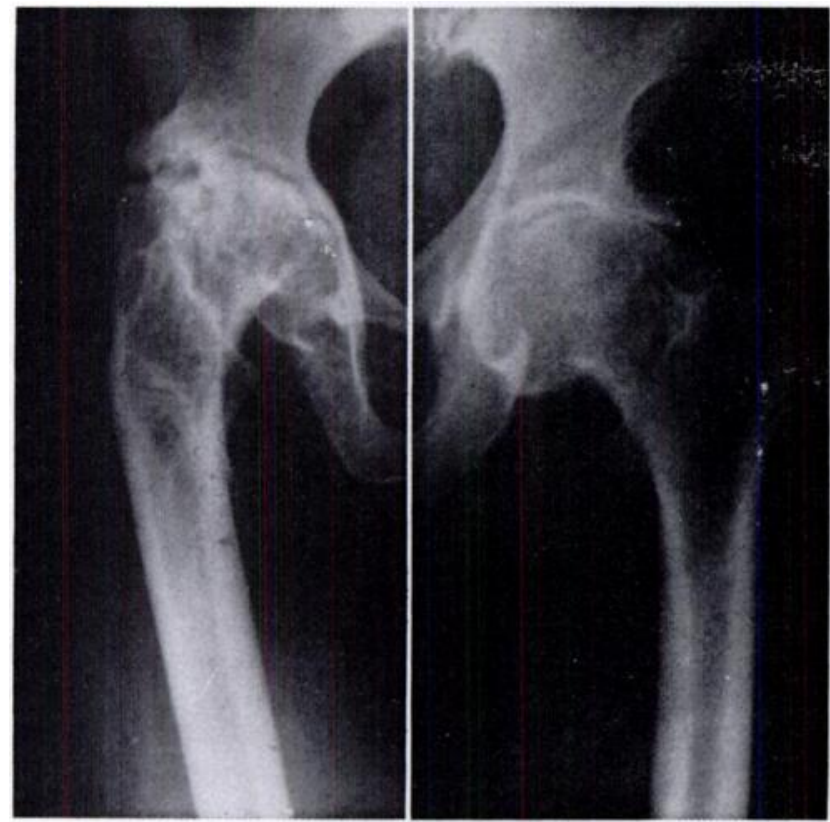

Fig. 5
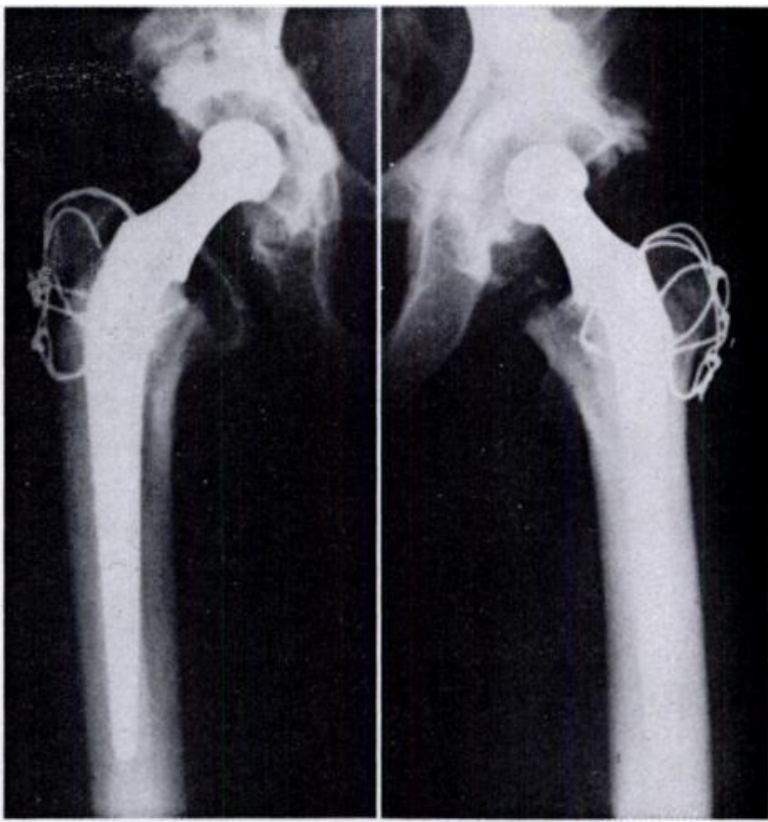

Fig. 6

Case 2. Figure 5-Radiograph of both hips before operation. Figure 6-After operation.

femoral head was negative for aerobic, anaerobic and tuberculous organisms. Histological examination showed no evidence of tuberculosis.

During the next few months her erythrocyte sedimentation rate remained between 40 and 60 millimetres in the first hour, although she was free of symptoms. Twelve months after operation a fluctuant mass appeared in the left thigh. This was explored and found to extend to the capsule of the hip. Cultures on separate occasions grew Staphylococcus aureus and Pseudomonas pyocyanea which were treated with gentamicin. Her erythrocyte sedimentation rate had risen to 82 millimetres. The radiograph is shown in Figure 2 . The wound continued to discharge and Mycobacterium tuberculosis was cultured. She was allergic to all antituberculous drugs, despite a course of desensitisation. Two years after operation a sinogram was performed (Fig. 3) and the prosthesis was removed (Fig. 4).

Case 2. This patient had developed tuberculosis of the right hip at the age of ten years and her hip had been immobilised in a frame for four years. At twenty-six years she had had an arthrodesis and was subsequently free of symptoms. She had a normal pregnancy at the age of thirty.

She presented at the age of fifty-one with a painful right hip, a valgus deformity of the right knee with instability and also a painful left hip. On examination, movements of the left hip were painful but normal. The right hip was fixed in 15 degrees of adduction and 20 degrees of flexion. The right knee had a valgus deformity of 30 degrees with laxity of the medial collateral ligament. There were scars of healed sinuses around the right hip, and the healed arthrodesis scar. Routine investigations recorded a haemoglobin level of 13.9 grams per 100 millilitres, a white cell count of 5400 per microlitre and an erythrocyte sedimentation rate of 12 millimetres in the first hour. Radiographs revealed calcified bilateral hilar nodes and an arthritic left hip, and suggested fibrous ankylosis of the right hip (Fig. 5).

In October 1976 she underwent a Charnley arthroplasty on the right hip, which proved to have fibrous ankylosis. Cultures taken at operation grew no pathogenic or tuberculous organisms; histological examination showed no tuberculosis. Recovery was uneventful but on two occasions (in the first and in the fourth week) the erythrocyte sedimentation rate was 56 and 42 respectively. Six weeks later she

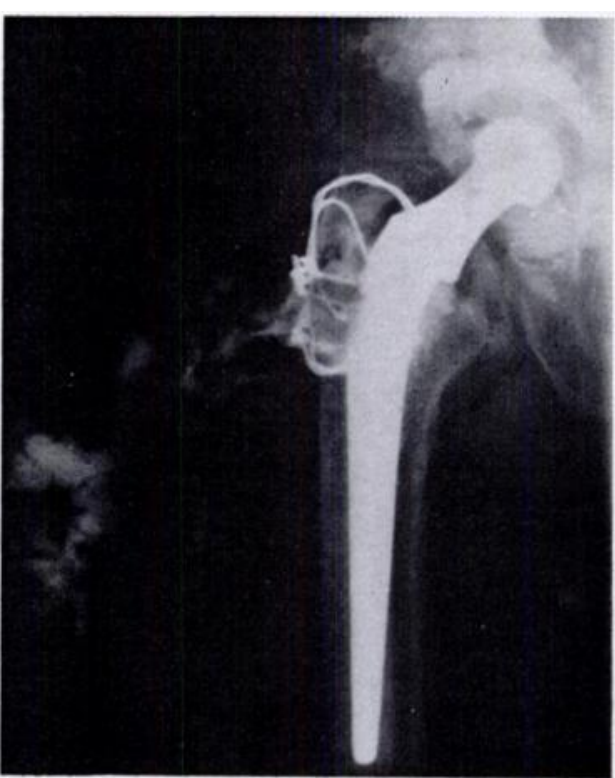

Fig. 7

Case 2. Sinogram fifteen months after replacement of the right hip.

underwent a Charnley arthroplasty on the left hip. Recovery was uneventful (Fig. 6). In November 1977, she underwent a supracondylar osteotomy of the right femur to correct the valgus deformity. Shortly after this, and thirteen months after the arthroplasty, she developed a sinus from the right hip from which Staphylococcus albus and later Mycobacterium tuberculosis were cultured. A sinogram was performed fifteen months later (Fig. 7). She is at present receiving rifampicin, isoniazid and ethambutol. Four months after starting this regime, the discharge is decreasing, but removal of the implants is likely to become necessary. 


\section{DISCUSSION}

These two cases have several similar features. Both patients were treated initially by traditional conservative immobilisation on a frame without antituberculous therapy. Although one of them had healed sinuses they both had been free of symptoms for many years and apparently free of infection for forty-two and thirtyseven years. They both also had fibrous ankylosis of the affected hip, which is known to be a hazardous condition especially as neither patient had had any antituberculous therapy.

The most successful treatment in primary tuberculosis of a joint is one that achieves sound bony union (Girdlestone and Somerville 1952) but even these cases must be reviewed critically since antituberculous therapy might not preclude reactivation. Hardinge et al. (1977) had no cases of reactivation after conversion of arthrodesed hips to arthroplasties. A search of the literature revealed only one vaguely similar case, that reported by McCullough (1977): his patient did not previously have tuberculosis of the hip but had radiographic evidence of calcified mesenteric glands, and seven years after a Ring prosthesis had been inserted tubercle bacilli were isolated from the wound, presumably from reactivation of the mesenteric glands. This highlights an important point that tuberculous organisms are much more likely to be the result of local reactivation, as in our cases. Although haematogenous spread is unlikely, careful scrutiny of the lung fields, the kidneys, the lymph nodes and other possible focal sites must be undertaken before operation, with special attention given to gastric washings and urine analyses. This is particularly important in communities where tuberculosis is still common.
The length of time of apparent freedom from active infection should not be a deciding factor as to whether to operate. As in our cases the infection had remained dormant for some forty years, it is presumed that the organisms may remain indefinitely. Histological and bacteriological sampling at operation from the capsule, acetabulum and femur is at best random and may prove negative, as it did in both our cases, in spite of dormant bacilli in other areas of the joint. Serial readings of erythrocyte sedimentation rate may be of little value: in one of our patients the levels were normal and in the other the level was elevated as a result of urinary infection. These facts make a strong case for routine prophylactic antituberculous therapy in all cases, with particular emphasis on those cases where the organisms are less likely to have been eradicated. In choosing a regime for prophylaxis, the less toxic agents such as para-aminosalicylic acid and isoniazid are preferable to streptomycin in combination with one of the second-line drugs such as rifampicin and ethambutol. We recommend that full dosage should be employed starting immediately before operation and continuing for at least twelve to eighteen months afterwards, depending to some extent upon serial readings of the erythrocyte sedimentation rate. This we think important since many more apparently healed tuberculous hips are being converted to arthroplasties, particularly in countries where tuberculosis is common. For patients with established infection, it is difficult to make firm recommendations. One of our patients is still undergoing antituberculous therapy and, although the sinus and discharge is improving slowly, the long-term outcome is uncertain. The other patient was allergic to treatment and, despite removal of the prosthesis, the wound continues to discharge.

\section{REFERENCES}

Girdlestone, G. R., and Somerville, E. W. (1952) Tuberculosis of Bone and Joint. Second edition. London: Oxford University Press. Hardinge, K., Williams, D., Etienne, A., MacKenzie, D., and Charnley, J. (1977) Conversion of fused hips to low friction arthroplasty. Journal of Bone and Joint Surgery, 59-B, 385-392.

McCullough, C. J. (1977) Tuberculosis as a late complication of total hip replacement. Acta Orthopaedica Scandinavica, 48, 508-510. 